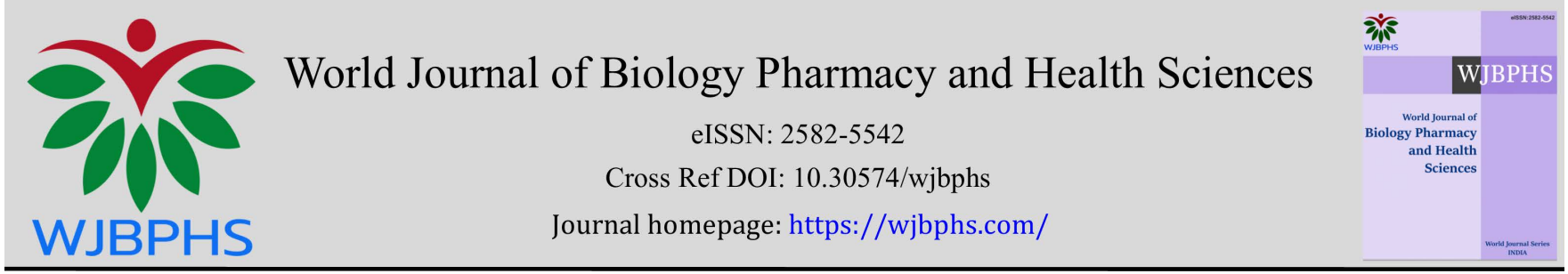

(RESEARCH ARTiCLE)

\title{
Cutaneous wound healing modulation of lesions infected by Staphylococcus epidermidis and treated with $4 \%$ stabilized sodium hypochlorite solution
}

Carolina Telini Rosa Rodrigues Alves ${ }^{1}$, Eduardo Monteiro Diniz Junqueira ${ }^{1}$, Camillo de Lellis Sandoval Filho ${ }^{1}$, Juliana Cruzera Antonio ${ }^{1}$, Melina Mizusaki Iyomasa-Pilon ${ }^{1}$, Helena Ribeiro Souza ${ }^{1,2}$ and Ana Paula Girol 1,2,*

${ }^{1}$ University Center Padre Albino (UNIFIPA), Catanduva, SP, Brazil (Department of Basics Sciences).

2 São Paulo State University, (UNESP), Institute of Biosciences, Humanities and Exact Sciences (IBILCE), São José do Rio

Preto Campus, SP, Brazil (Department of Biology, Laboratory of Immunomorphology).

World Journal of Biology Pharmacy and Health Sciences, 2021, 07(02), 032-042

Publication history: Received on 17 June 2021; revised on 01 August 2021; accepted on 03 August 2021

Article DOI: https://doi.org/10.30574/wjbphs.2021.7.2.0077

\begin{abstract}
Background: Stabilized sodium hypochlorite solution at $4 \%(4 \% \mathrm{NaClO})$ is a product with healing properties but still little studied scientifically. Here we evaluated the administration of $4 \% \mathrm{NaClO}$ in a model of clean and infected skin lesions.

Methods: Lesions were obtained on the dorsal region of Wistar rats. The wounds of some animals were inoculated with Staphylococcus epidermidis (50uL, 10-7) and clean and infected lesions were treated with Trofodermin ${ }^{\circ}$ or $4 \% \mathrm{NaClO}$ (diluted at $0.4 \%$ ) as the following groups: Trofodermin®; S. epidermidis + Trofodermin®; 4\% NaClO; S. epidermidis + $4 \% \mathrm{NaClO}$. Wounds sizes were daily measured and the excision of lesions was performed on day 8 for histopathological evaluation and immunohistochemical studies of Annexin A1 (AnxA1), receptors for formylated peptides (FPR)1 and FPR2 and vascular endothelial growth factor (VEGF). Levels of interleukin (IL)-1 $\beta$ and tumor necrosis factor (TNF)- $\alpha$ in the supernatants of the macerated lesions were also measured.
\end{abstract}

Results: Wounds treated with 4\% NaClO closed faster than those treated with Trofodermin $\circledast$, especially in the infected group. 4\% NaClO reduced the expressions of AnxA1, FPR1, FPR2 and VEGF, as well as decreased the levels of IL-1 $\beta$.

Conclusion: These results pointed the potential of $4 \% \mathrm{NaClO}$ in wound healing which opens the possibility for new therapeutic strategies.

Keywords: Wound healing; NaClO; AnxA1; FPR; VEGF

\footnotetext{
* Corresponding author: Ana Paula Girol

University Center Padre Albino (UNIFIPA), Catanduva, SP, Brazil (Department of Basics Sciences).

Copyright $(2021$ Author(s) retain the copyright of this article. This article is published under the terms of the Creative Commons Attribution Liscense 4.0.
} 


\section{Graphical extract:}

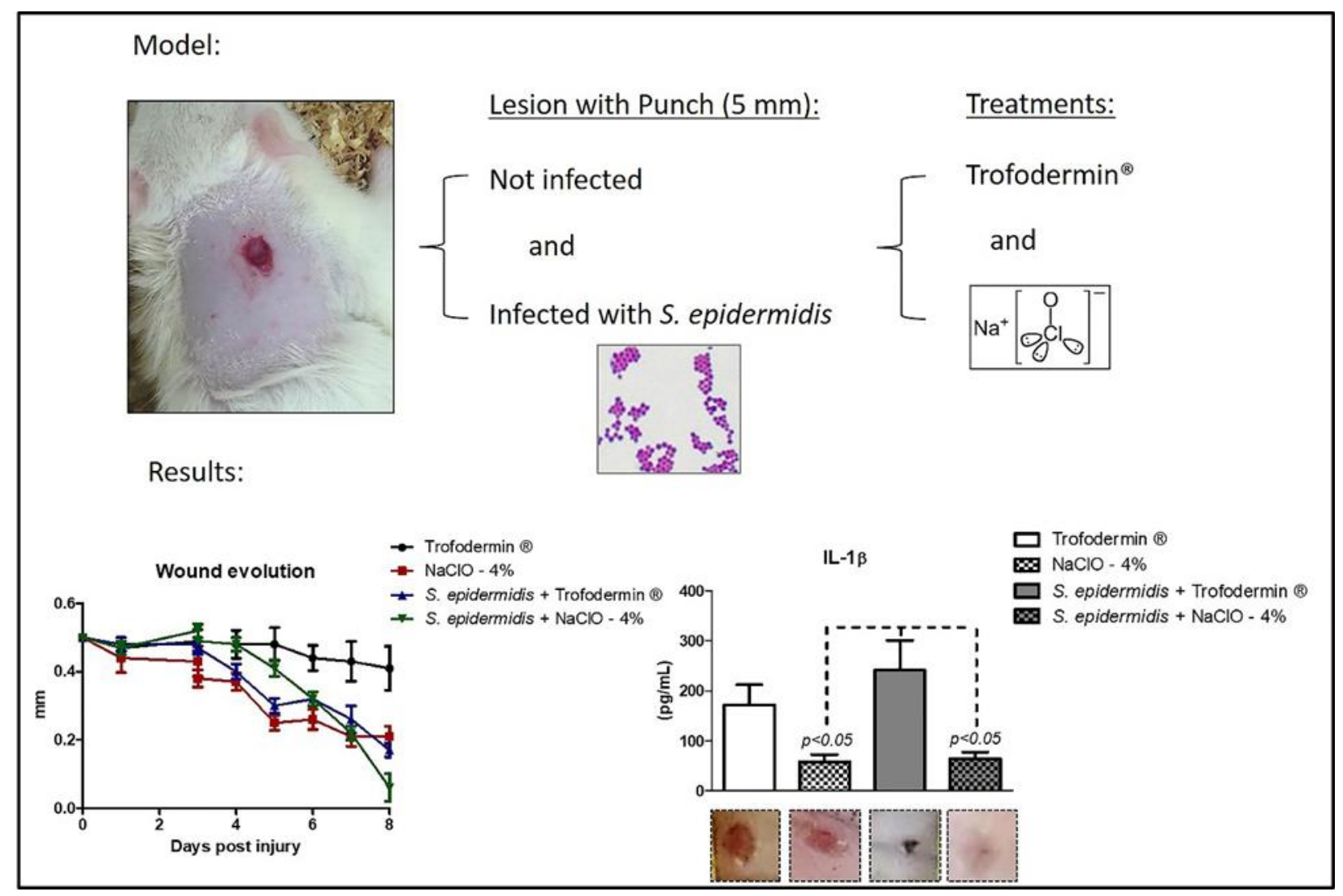

\section{Introduction}

The skin healing involves a cascade of events that promotes wound healing and restores the anatomy of the skin. The healing process is summarized in the phases of inflammation, proliferation and maturation (1). Several cells orchestrate these phases and act through the release of proinflammatory factors, such as tumor necrosis factor- $\alpha$ (TNF- $\alpha$ ), interleukin-1 $\beta$ (IL-1 $\beta$ ) and anti-inflammatory mediators like the Annexin A1 protein (AnxA1), related to the resolution of the inflammation (2-4). In the tissue regeneration process, the growth factors such as the vascular endothelial growth factor (VEGF) promote cell proliferation and differentiation. These factors restore blood flow to tissues and are essential for granulation tissue formation after an injury (5).

The AnxA1 protein is a member of the annexin superfamily that exhibits affinity for $\mathrm{Ca}^{++}$and presents an unique $\mathrm{N}$ terminal portion for each member of the superfamily, which gives them specificity of action ( 3 ). In recent years, the AnxA1 protein has been related to reparative and antifibrotic functions by regulating the fibroblast activity $(2,4,6-10)$. AnxA1 can act in an autocrine and paracrine way by binding with its formylated peptide receptors (FPR) 1 and 2, from the class of G-protein-coupled receptors expressed by different cell types, including leukocytes, endothelial and epithelial cells (3).

Although it is vital to the body, the healing process can lead to pathological scars $(4,11)$. Therefore, laboratory models are developed for the study of the healing process, for example, by cuts or excisions of the skin (12). Models of wounds mainly infected by bacteria were also proposed for testing new therapies (13), since the infection by microorganisms is one of the risk factors in patients.

In this context, chlorinated products like sodium hypochlorite $(\mathrm{NaClO})$ is proven to be microbiocidal for disinfection of surfaces (14-16) and in addition they have shown therapeutic applicability, as dental root canal irrigants (17), skin decontamination $(18,19)$ and wound healing $(20-23)$.

Previous investigations showed that diluted solutions of sodium hypochlorite decontaminated human cadaveric skin colonized by Staphylococcus aureus, Pseudomonas aeruginosa or Candida albicans with no significant effect on basal cell viabilities $(18,19)$. A research compared 3 topical treatments against spontaneous ulcerative dermatitis in C57BL/6 
mice and indicated better healing in the group treated with hypochlorite compared to the others treated with tripleantibiotic ointment or povidone-iodine and sulfadiazine (21).

Other investigation pointed that human fibroblasts were viable after exposure to serial dilutions of unbuffered sodium hypochlorite solutions, but the same dilutions of unbuffered sodium hypochlorite were bacterical to Pseudomonas aeruginosa, Staphylococcus aureus and Streptococcus pyogenes (20).

Sodium hypochlorite ( $\mathrm{NaClO}$ ) wound irrigation solutions have gained increasing attention in daily clinical practice in the prevention and treatment of low-level wound infections due to the lower cytotoxicity (24). Recently, in a case report the administration of the hypochlorous acid to irrigate a horseshoe perianal abscess showed rapid fever subside and wound closure, suggesting that this solution was able to adequately destroy the infecting microorganisms (23).

The 4\% stabilized sodium hypochlorite solution ( $\mathrm{NaClO}$ - 4\%) has been applied in empirical use in veterinary clinics in Brazil for its healing properties. Thus, the aim of this study was to evaluate the wound healing and microbiocidal properties of $4 \% \mathrm{NaClO}$ in induced wounds in the skin of rats, infected or not with Staphylococcus epidermidis, in an attempt to reproduce and scientifically verify the method used in veterinary clinics, on the used dilution and administration of the product.

\section{Material and methods}

\subsection{Animals}

Adult female Wistar rats (250-300 g) were obtained from the University Center Padre Albino (UNIFIPA) Catanduva, SP, Brazil and divided into four groups ( $n=5$ / group). The animals were kept in individual cages in a controlled environment (24 to $25^{\circ} \mathrm{C}$ ) with water and food ad libitum. All experimental procedures were conducted in accordance with EU Directive 2010/63/EU for animal experiments, the guidelines for biomedical research stated by the Brazilian Societies of Experimental Biology and were approved by the Ethics Committees on Animal Use at UNIFIPA (Certificate No 04/18), Brazil. Also, all experiments were performed in accordance with the National Institutes of Health (NIH). The experiments were designed to minimize the number of animals used and their suffering during the execution of the protocols and all animals were evaluated by the veterinarian of the institution.

\subsection{Wound model and treatment protocols}

The animals were anesthetized intraperitoneally with $0.2 \mathrm{~mL} / 100 \mathrm{~g}$ ketamine and 0,05mL/100g xylazine, then, subjected to shaving and asepsis in the dorsal area to induce a lesion of $5 \mathrm{~mm}$ of diameter with a metal Punch. The animals were given codeine $(1 \mathrm{~mL} / \mathrm{kg})$ by gavage immediately after lesion induction and, during the following days, the analgesic was provided in water $(30 \mathrm{mg} / \mathrm{L})$.

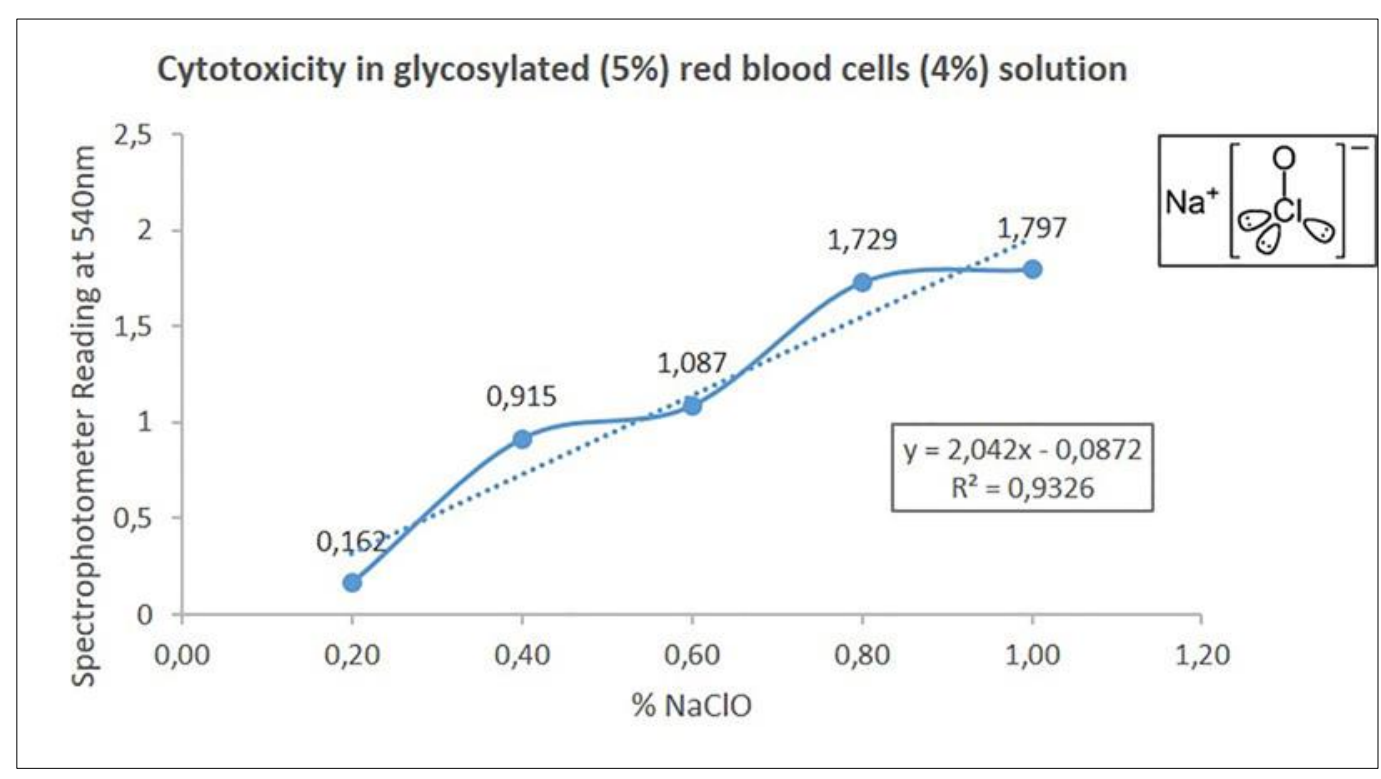

Figure 1 Cytotoxicity analysis. Analysis of 4\% NaClO cytotoxicity through hemolysis 
Two groups of animals had their wounds infected with S. epidermidis bacteria (Gram +), one of the most commonly found microorganisms in skin disorders (25), by the inoculation of $50 \mathrm{uL}$ of solution of the bacterial culture medium at a concentration of $10^{-7} \mathrm{CFU} / \mathrm{mL}(26)$.

The clean and infected lesions were treated from day 1 of induction with Trofodermin® ointment (Pfizer, NY, USA), which is considered a standard treatment, or $4 \% \mathrm{NaClO}$ (Chemical Dioxide, SP, Brazil), setting the treatment groups:

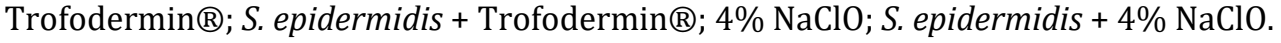

Prior administration, the product cytotoxicity was assessed in an in vitro hemolysis test. Different concentrations of $4 \%$ $\mathrm{NaClO}$ diluted in physiological saline $(0.2 ; 0.4 ; 0.6 ; 0.8 ; 1.0)$ were mixed with a glycosylated solution $(5 \%)$ of human blood (4\%). The absorbance was read in a spectrophotometer (540 nm) (Figure 1). The concentration of $0.4 \%$ showed only relative cytotoxicity and it was selected to be sprayed on the wounds of the animals in this experiment because it is the empirically concentration used in veterinary clinics (Figure 2).

Eight days after the injury, the animals were euthanized by overdose of anesthetic (isoflurane) for excision of lesions.
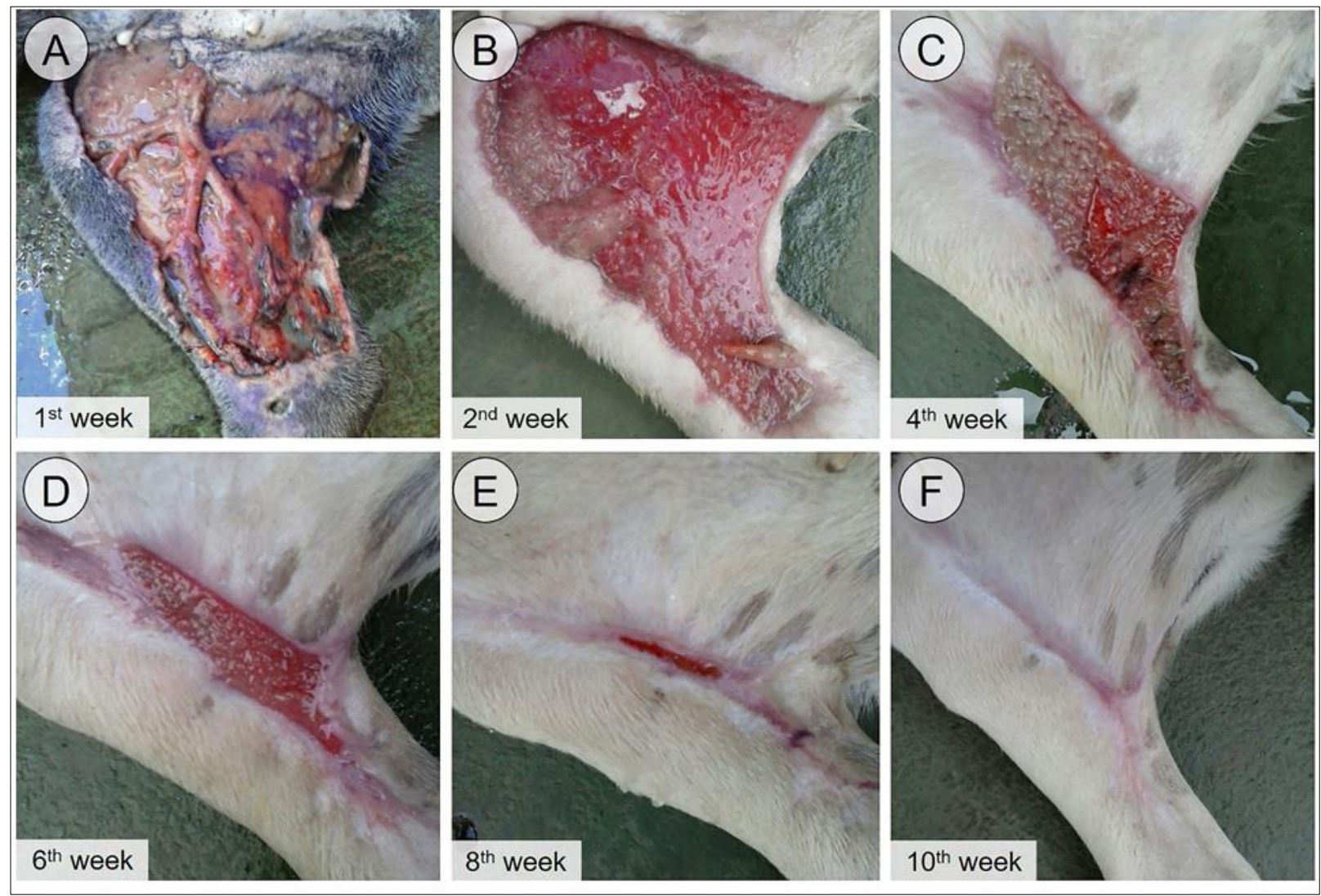

Figure 2 Evolution of wound healing with daily application of $4 \% \mathrm{NaClO}$

To treat the extensive injuries caused by running over a dog, found abandoned on a farm. Daily applications of $4 \% \mathrm{NaClO}$ (diluted to $0.4 \%$ ) were used as an experimental resource. A broad-spectrum antibiotic (Benzylpenicillin Procaine 15,000,000 IU, Benzylpenicillin Benzatin 10,000,000 IU, Dihydroestreptomycin 34.12 g, Vehicle qsp $100 \mathrm{~mL}$ ) was injected on the 1 st day of admission to the veterinary clinic ( $1 \mathrm{~mL} / \mathrm{kg}$ in a single dose).

\subsection{Morphological, histopathological and immunohistochemical studies}

The wounds of all animals were daily photographed and measured in order to monitor the wound healing process in the different groups.

After euthanasia, lesion fragments were fixed in $4 \%$ formalin, processed for inclusion in paraffin and sectioned ( $4 \mu \mathrm{m})$. The process of skin healing was evaluated histologically by hematoxylin-eosin staining (HE). 
FPR1, FPR2 and VEGF were detected by immunohistochemistry. The sections underwent antigen retrieval and endogenous peroxidase activity blocking and were incubated for 3 hours, in a moist chamber at room temperature, with the following primary antibodies diluted in 1\% BSA: Rabbit anti-AnxA1 (Invitrogen, No. 71-3400, polyclonal, 1:600); Rabbit anti-VEGF (Abcam, ab46154, polyclonal, 1:100); anti-FPR1 (Biorbyt, orb13410, 1:400) and anti-FPR2 (Abcam, ab203129, 1:400).

The sections were then incubated with the biotinylated secondary antibody (Histostain Kit, Invitrogen) and immersed in a conjugated streptavidin-peroxidase complex. The substrate diaminobenzidine (DAB Kit, Invitrogen) was used for the development and, thereafter, the sections were stained with Hematoxylin.

For densitometric analysis, 20 different points of the epidermis and dermis in 5 different fields per slide were evaluated to obtain an average related to the intensity of immunostaining. The values were obtained as arbitrary units (0 to 255) by Leica image analyzer Image Analysis software (4).

\subsection{Cytokines Levels}

Fragments of lesions of all groups were macerated in liquid nitrogen and placed in Eppendorf tubes, in which $500 \mu \mathrm{L}$ of a solution containing protease inhibitors (Mini Complete EDTA-free, Roche Applied Science, Mannheim, Germany) and phosphatase (PhosSTOP, Roche Applied Science, Mannheim, Germany) were added. The material was incubated for 1 hour at $4{ }^{\circ} \mathrm{C}$ under constant stirring, centrifuged at $14.000 \mathrm{rpm}$ for 10 minutes at $4{ }^{\circ} \mathrm{C}$ and then the supernatants were collected and immediately frozen at $-70^{\circ} \mathrm{C}$.

IL-1 $\beta$ and TNF- $\alpha$ levels were quantified in the supernatants of macerated lesions using the rat cytokine MILLIPLEX MAP Kit (RECYTMAG-65K; Millipore Corporation, USA) and analyzed on LUMINEX xMAP MAGPIX equipment (Millipore Corporation, USA). The concentration of the analytes was determined by MAGPIX xPONENT software (Millipore Corporation, USA). Results were expressed as mean \pm standard error of the mean (S.E.M.) of cytokines concentrations $(\mathrm{pg} / \mathrm{mL})$.

\subsection{Statistical analysis}

The results were previously submitted to a descriptive analysis and determination of normality. As the samples were normally distributed, the Analysis of Variance (ANOVA) one way was used, followed by the Bonferroni test. All values were expressed as mean \pm SEM. P values $<0.05$ were considered statistically significant.

\section{Results}

\subsection{The treatment with $4 \% \mathrm{NaClO}$ was effective in wound healing}

The morphological evaluations indicated that the lesions healed faster in the groups treated with $4 \% \mathrm{NaClO}$ (diluted at $0.4 \%$ ) compared to animals treated with Trofodermin ${ }^{\circledR}$, especially in the group with infected lesions (Figure 3E). These findings were corroborated by histopathological analysis that also showed faster reepithelization, rearrangement of the dermis and reappearance of epithelial attachments by treatment with $4 \% \mathrm{NaClO}(0.4 \%)$ compared to the groups treated with Trofodermin $®$ (Figure 3A-D).

\subsection{AnxA1, FPR1, FPR2 and VEGF expressions were decreased by treatment with $4 \%$ NaClO (diluted at $0.4 \%$ )}

Immunohistochemical studies showed expression of the protein AnxA1 in the epidermis and dermis of the lesions (Figure 4). By densitometric evaluation, the immunostaining of this anti-inflammatory protein was decreased in the epidermis of the 4\% $\mathrm{NaClO}$ (0.4\%)-treated-uninfected-group (Figure 4B and E). FPR1 and FPR2 receptors (Figure 4) were expressed in the same regions observed for AnxA1. FPR1 immunostaining was significantly reduced in the dermis of the 4\%-NaClO-treated-groups, mainly in the animals with infected lesions (Figure 5B). Differently, FPR2 expression was decreased in both epidermis (Figure 5C) and dermis (Figure 5D), especially in the S. epidermidis $+4 \% \mathrm{NaClO}$ group.

The expression of VEGF (Figure 6) was studied in the dermis and showed a significant reduction promoted by the treatment with 4\% $\mathrm{NaClO}(0.4 \%)$ (Figure 6E) in the infected (Figure 6D) and uninfected (Figure 6B) groups. The specificity of the immunostaining was confirmed by the reaction controls (Figures 4G and 6F). 


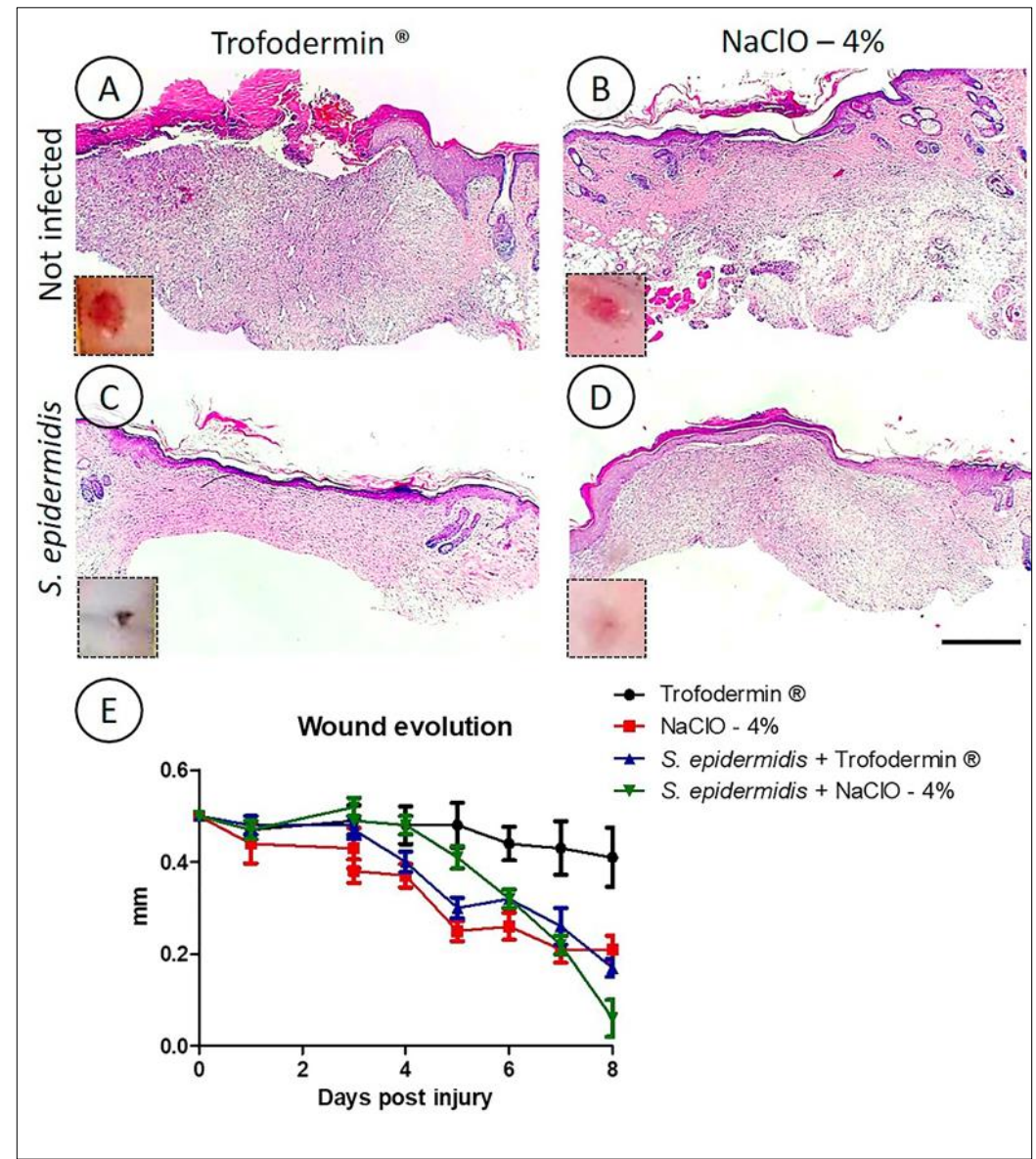

Figure 3 Morphological and histopathological evaluation of the lesions. Trofodermin® (A); 4\% NaClO (B); S. epidermidis + Trofodermin $®(C) ;$ S. epidermidis + NaClO - 4\% (D) groups, 8 days after the lesion. Daily measurements of the lesions in the different groups, indicating faster healing and reepithelialization in the groups treated with $4 \% \mathrm{NaClO}$ (E). Hematoxylin Eosin staining. $200 \mu \mathrm{m}$ bar

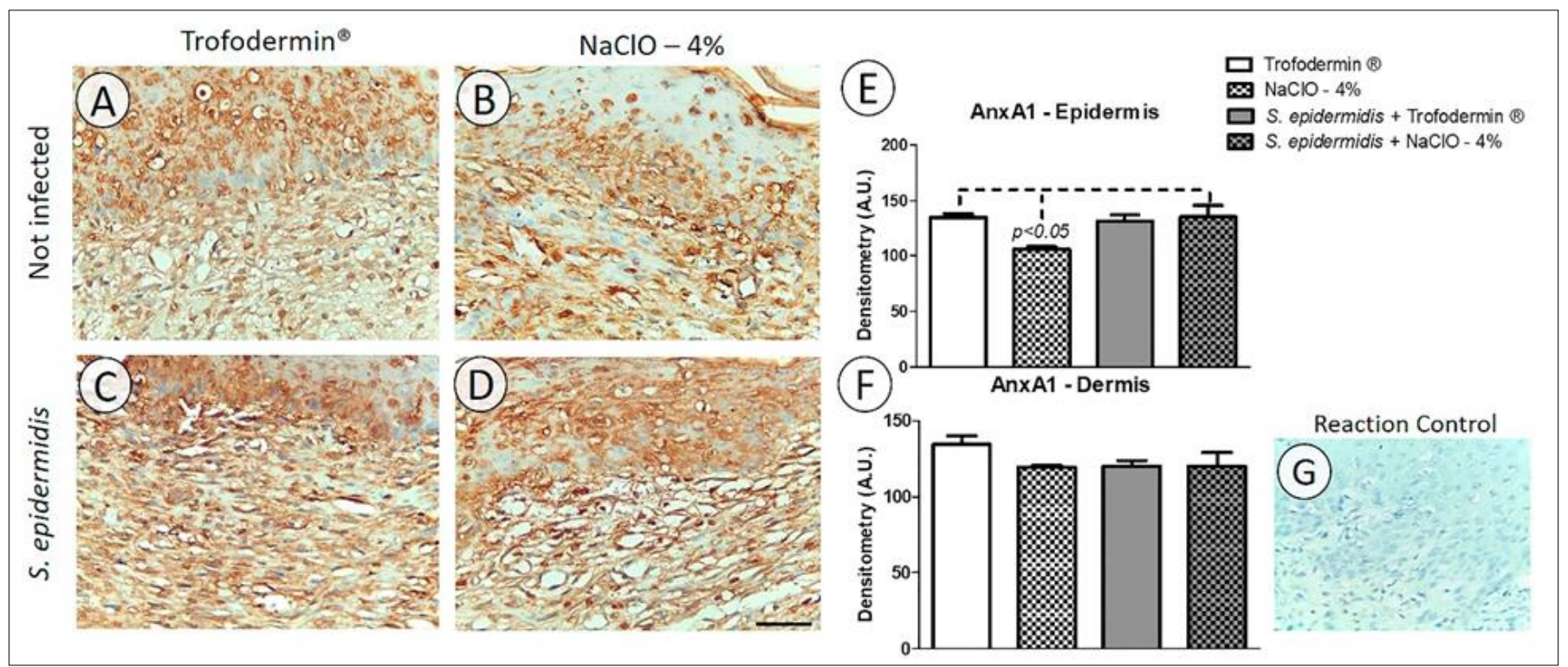

Figure 4 AnxA1 expression in the healing process. AnxA1 expression in the epidermis and dermis of the Trofodermin ${ }^{\circ}$ (A); 4\% NaClO (B); S. epidermidis + Trofodermin $\AA$ (C); S. epidermidis + NaClO - 4\% (D) groups. Counterstaining: Hematoxilin. $50 \mu \mathrm{m}$ bars. Reaction control (G). Densitometric analysis. In the epidermis (E) and dermis (F). The data are represented as mean \pm standard error of the mean (S.E.M), ( $n=5$ / group) 


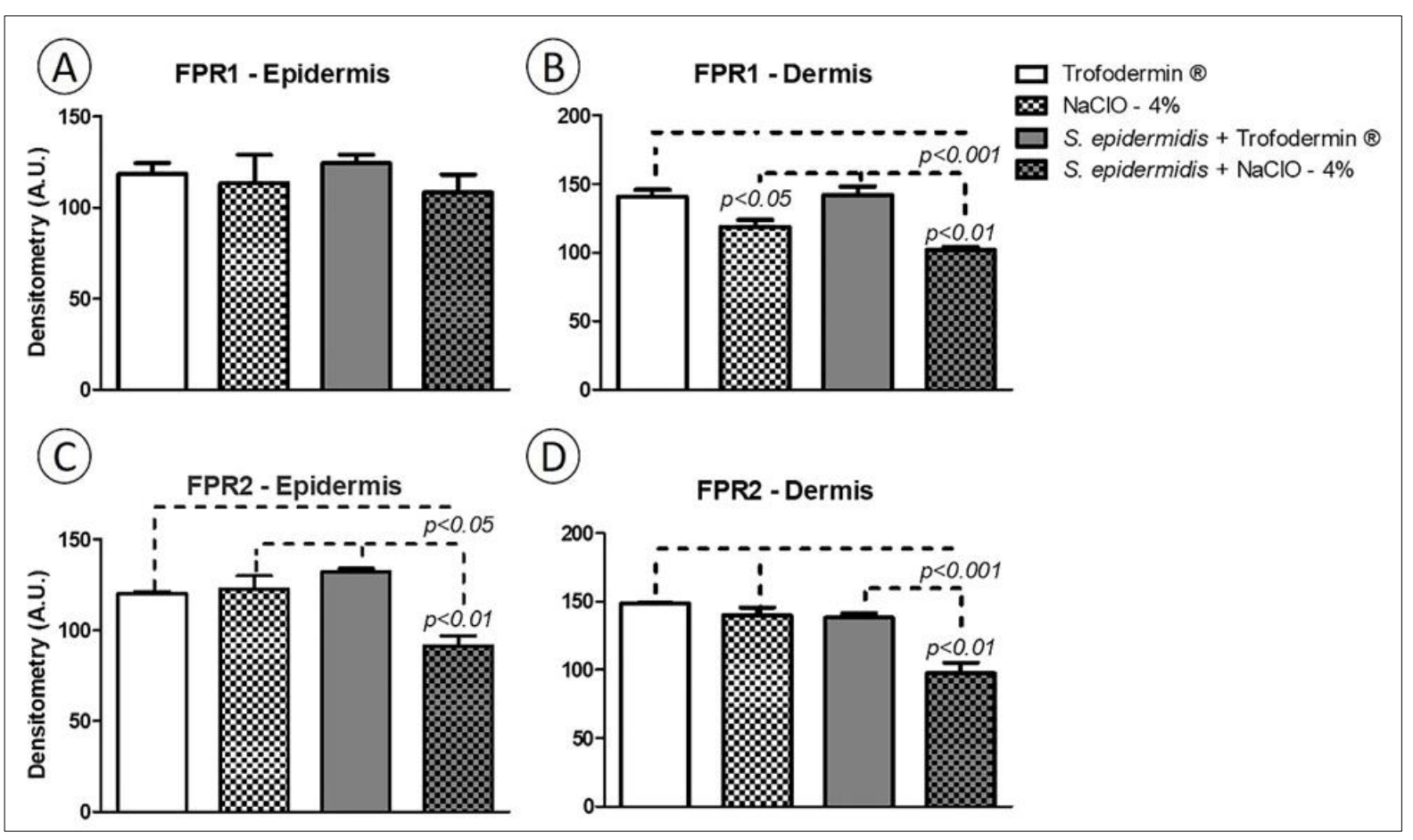

Figure 5 Expression of FPR receptors in the healing process. Densitometric analysis of FPR1 (A and B) and FPR2 expressions (C and D), respectively in the epidermis and dermis in the Trofodermin $₫$; S. epidermidis + Trofodermin $\AA$; $4 \% \mathrm{NaClO}$; S. epidermidis $+4 \% \mathrm{NaClO}$ groups. The data are represented as mean \pm standard error of the mean (S.E.M), (n=5 / group)

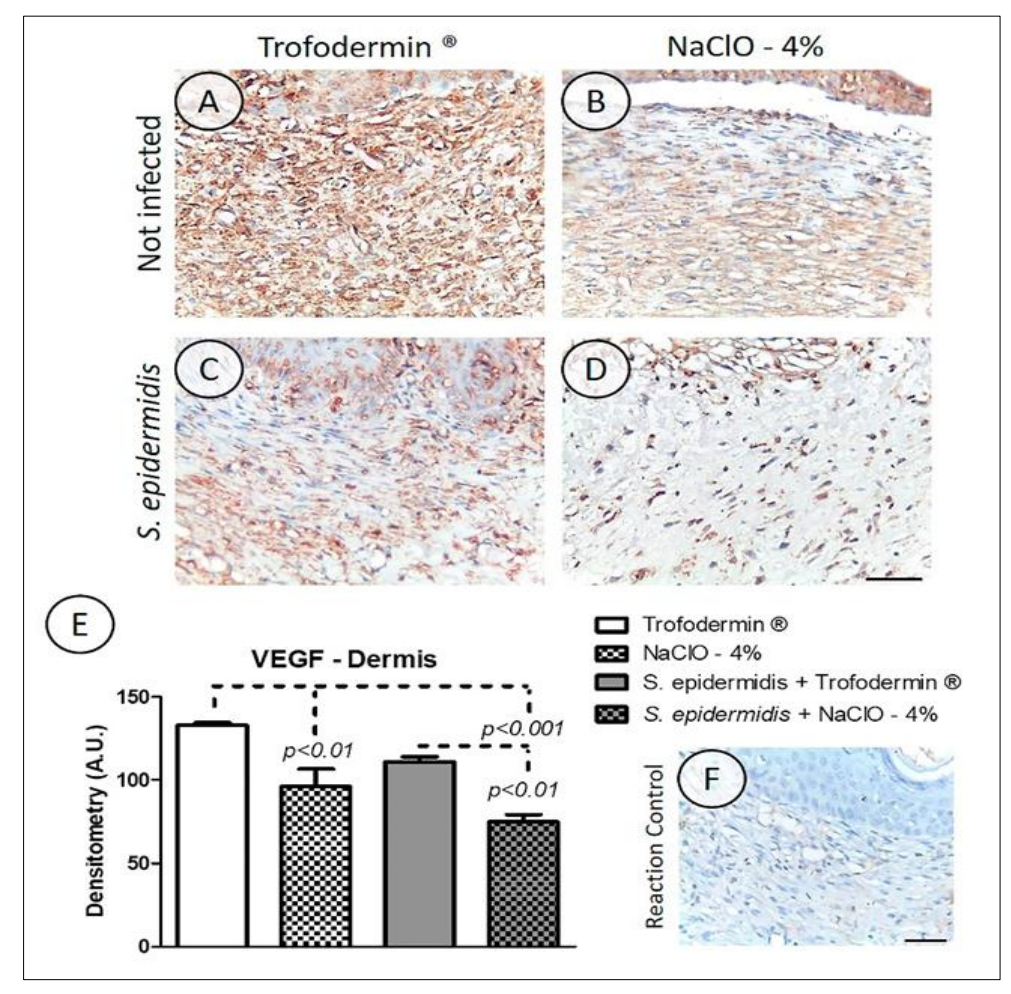

Figure 6 VEGF expression in the dermis of the lesions. Trofodermin $₫(A) ; 4 \% \mathrm{NaClO}$ (B); S. epidermidis + Trofodermin ${ }^{\circ}$ (C); S. epidermidis + 4\% NaClO -(D) groups. Counterstaining: hematoxilin. $50 \mu \mathrm{m}$ bars. Absence of immunoreactivity in the reaction control (F). Densitometric analysis. (E) The data are represented as mean \pm standard error of the mean (S.E.M), ( $n=5$ / group) 


\subsection{Reduced levels of pro-inflammatory cytokines by $4 \% \mathrm{NaClO}$ (diluted at $0.4 \%$ ) administration}

Similar to the immunohistochemistry results found for the expression of the proteins, the levels of the IL- $1 \beta$ (Figure 7A) and TNF- $\alpha$ (Figure 7B) cytokines were also decreased with the treatment with $4 \%$ NaClO $(0.4 \%)$, particularly for IL- $1 \beta$ $(\mathrm{p}<0.05)$

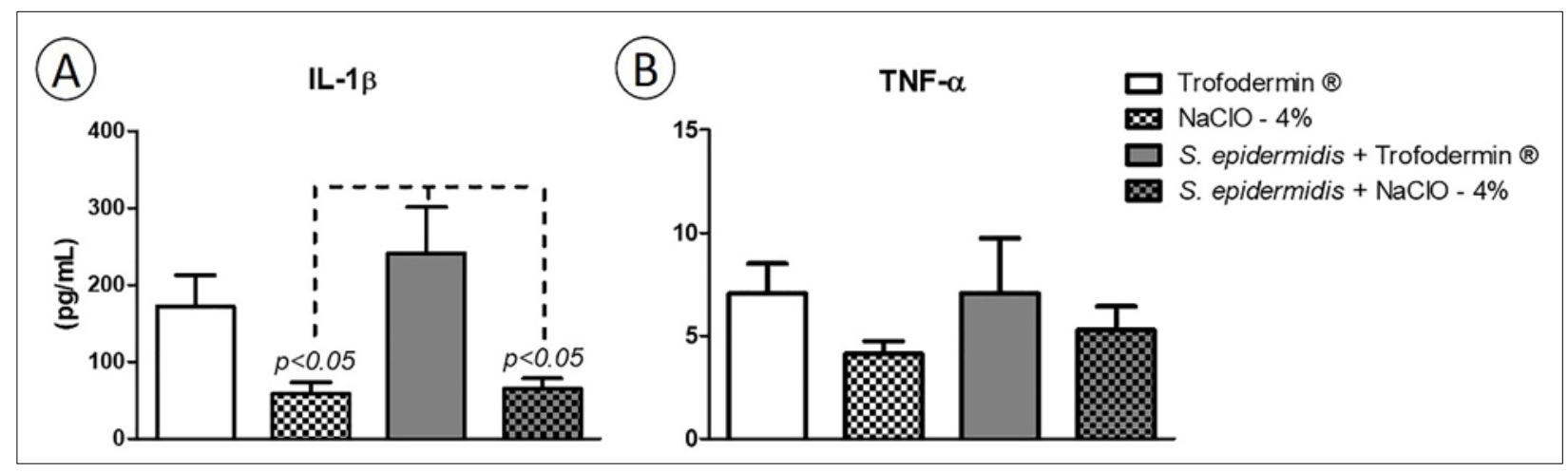

Figure 7 Levels of cytokines in the lesion supernatants. Interleukin (IL)-1 $\beta$ (A) and tumor necrosis factor (TNF)- $\alpha$ (B). Trofodermin $\AA ;$ S. epidermidis + Trofodermin $® ; 4 \% \mathrm{NaClO} ;$ S. epidermidis + 4\% NaClO groups. The data are represented as mean \pm standard error of the mean (S.E.M), ( $n=5 /$ group)

\section{Discussion}

In the present study, our group investigated the antibacterial and healing properties of the compound $4 \% \mathrm{NaClO}$ (diluted at $0.4 \%$ ) in lesions infected or not with S. epidermidis. First we analyzed all the lesions morphologically and we found faster healing in the $4 \% \mathrm{NaClO}$-treated groups, especially in the infected one.

$\mathrm{NaClO}$ has a broad antimicrobial spectrum and rapid bactericidal action $(15,16)$. The effect of $0.04 \% \mathrm{NaOCl}$ was shown in maintaining the viability of remaining pulp cells in the revascularization of immature permanent teeth with apical periodontitis while maintaining desirable antibacterial properties (27). Topical antimicrobials are one component in burn wound care, because they suppress microbial growth and promote the balance in favor of keratinocytes and wound healing (22). An unbuffered solution of $\mathrm{NaClO}$ with a concentration of $0.006 \%$ may be suitable for the topical management of burn wound infections caused by common pathogens (20). These authors indicated that $\mathrm{NaClO}$ at a concentration of $0.006 \%$ was bactericidal to Pseudomonas aeruginosa, Staphylococcus aureus and Streptococcus pyogenes but left 88\% fibroblasts viable. Also the use of hypochlorous acid in an immunocompromised patient with a horseshoe perianal abscess was proved to be better than diluted povidone-iodine to lavage the wounds. The irrigation with hypochlorous acid was less painful and rapidly subside fever. The infection cleared within 2 weeks and final wound closure was achieved after 6 weeks compared while in the irrigation with diluted povidone-iodine the final wound closure was after 10 weeks (23). Therefore, the antimicrobial activity of $\mathrm{NaClO}$ may have helped to prevent the development of $S$. epidermidis and inflammation as well as promoted the healing process in our study.

Continuing the research, the immunostaining of AnxA1 protein and its receptors, FPR1 and FPR2, which are related to anti-inflammatory and reparative functions, was evaluated. In the groups treated with $\mathrm{NaClO}-4 \%$, it was observed a reduction in the expression of AnxA1 and FPRs, showing a smaller magnitude of the inflammatory process and a better tissue recovery capacity compared to the group treated with the standard ointment. Importantly, the uninfected group treated with $\mathrm{NaClO}-4 \%(0.4 \%)$, at the time of these analyzes, eight days after injury, had already showed scar tissue and neogenesis of epithelial appendages. AnxA1 is an anti-inflammatory protein and is involved in several biological processes such as proliferation and cell death (3), which could explain its lower expression in the groups in which the wound healing occurred earlier. AnxA1 has also been related to antifibrotic and healing function in mucosal and skin lesion models $(4,12,28)$. Thus, the group treated with $\mathrm{NaClO}-4 \%$ also had the advantage of faster tissue repair and lower degree of fibrosis after treatment.

The role of AnxA1 in intestinal wound healing with participation of FPR1 and reduction of inflammation was evidenced in experimental colitis in rats (28). In a study about induced pulmonary fibrosis, it was observed a greater deposition of collagen in the lung of knockout mice for AnxA1 (9). In another model study on pulmonary fibrosis, the destruction of lung architecture, increased inflammation and collagen accumulation were found in AnxA1 knockout mice (7). Another investigation showed an increased expression of AnxA1 and FPR2 in the animals with fibrotic nephropathy 
compared to their controls (10). Studies on models of cutaneous lesions showed the relation of AnxA1 and skin healing. One of them pointed out that in the skin incisions treated with the mimetic peptide of AnxA1 the wound healing occurred faster (12). In another investigation that was performed by our research group, it was observed higher AnxA1 expression in skin scars of burns treated with standard commercial ointment and better healed compared to the untreated group (4).

In relation to FPRs, these receptors are present in innate immunity cells, fibroblasts, endothelial and epithelial cells $(29,30)$ where they act as pattern recognition receptors (PRRs) recognizing thousands of types of bacterial peptides (Pathogen-Associated Molecular Patterns - PAMPs). The binding of the FPR to their agonists is related to the immune response after tissue injuries (22), since in knockout mice for FPR1 and FPR2 wound healing is slower (31). In this work, we observed a reduction of FPR1 expression in the dermis while FPR2-immunostaining was decreased in the dermis and epidermis of animals infected with $S$. epidermidis and treated with $4 \% \mathrm{NaClO}(0.4 \%)$, suggesting an important microbicidal activity of the tested compound. Among the FPRs analyzed in this study, FPR2 had a more reduced expression after the treatment with $4 \% \mathrm{NaClO}(0.4 \%)$ in infected wounds, showing greater involvement of this receptor. The FPR1 recognizes a wide range of small bacterial peptides, while FPR2 is capable of binding to larger peptides and, mainly, those derived from Staphylococcus (staphylococcal-derived phenol-soluble modulins) (30), the kind of bacteria used in our model.

Confirming the data from the other immunostainings, VEGF expression was also reduced in animals treated with $4 \%$ $\mathrm{NaClO}(0.4 \%)$, especially in the infected group. This growth factor is related to the initial phases of the healing process when occur the formation of the granulation tissue (32). However, VEGF expression has also been found in more advanced phases, on 7 days after the burn injury in mice (33) and rats $(33,34)$. Despite the increased expression of growth factors, which indicate good healing (35), the $\mathrm{NaClO}+$ S. epidermidis group had closed lesions at the time of biopsy, while Trofodermin $®$ group had open wounds, which may be explain the VEGF reduction in the groups treated with $4 \% \mathrm{NaClO}(0.4 \%)$ compared to standard treatment.

In complementary studies, we observed lower levels of IL- $1 \beta$ and TNF- $\alpha$ cytokines in the $4 \%$ NaClO $(0.4 \%)$-treatedgroups, with a significant reduction in IL-1 $\beta$. Both cytokines are related to inflammatory and reepithelialization processes and they are released by leukocytes, mainly overexpressed in the early stages of healing $(4,32,33)$. The reduction of the proinflammatory cytokines may be considered as a parameter for wound treatment; thus, the tested treatment showed efficacy. In ulcerative dermatitis in mice, once-daily topical treatment with $\mathrm{NaClO} 0.005 \%$ prevented further tissue damage by inhibiting excessive neutrophilic oxidative burst and, then, allowed tissue healing (21). Also, topical hypochlorite ameliorates NF-kB-mediated skin diseases in mice (36). Our results corroborate these works and point to the therapeutic wound healing potential of $\mathrm{NaClO}$ with protective effect against infection.

\section{Conclusion}

Our analysis showed faster healing in rats infected with the bacterium S. epidermidis and treated with $4 \% \mathrm{NaClO}$ (diluted at $0.4 \%$ ) through measurements of lesion size, histological results, immunohistochemical studies of AnxA1, FPR1, FPR2 and VEGF as well as the quantification of pro-inflammatory cytokines. These data indicate that $4 \% \mathrm{NaClO}(0.4 \%)$ has healing properties and important microbicide profile and supports its therapeutic application.

\section{Compliance with ethical standards}

\section{Acknowledgments}

We want to acknowledge to Dr. Roberto Pucci Rodrigues Alves, to provide the photos of the Supplementary material and to provide the $4 \%$ stabilized sodium hypochlorite solution for our experiments.

\section{Disclosure of conflict of interest}

The authors declare that there is no conflict of interest.

\section{Statement of ethical approval}

All experimental procedures were conducted in accordance with EU Directive 2010/63/EU for animal experiments, the guidelines for biomedical research stated by the Brazilian Societies of Experimental Biology and were approved by the Ethics Committees on Animal Use at UNIFIPA (Certificate No 04/18), Brazil. 


\section{References}

[1] Takeo M, Lee W, Ito W. Wound Healing and Skin Regeneration. Cold Spring Harb Perspect Med. 2015; 5(1).

[2] Leoni G, Neumann P-A, Sumagin R, Denning TL, Nusrat A. Wound repair: role of immune-epithelial interactions. Mucosal Immunol. 2015; 8(5): 959-68.

[3] Gobbetti T, Cooray SN. Annexin A1 and resolution of inflammation: Tissue repairing properties and signalling signature. Biol Chem. 2016; 397(10): 981-93.

[4] Souza HR, De Azevedo LR, Possebon L, De Souza Costa S, Iyomasa-Pilon MM, Oliani SM, et al. Heterogeneity of mast cells and expression of Annexin A1 protein in a second degree burn model with silver sulfadiazine treatment. PLoS One. 2017; 12(3): 1-17.

[5] Tang QL, Han SS, Feng J, Di JQ, Qin WX, Fu J, et al. Moist exposed burn ointment promotes cutaneous excisional wound healing in rats involving VEGF and bFGF. Mol Med Rep. 2014; 9(4): 1277-82.

[6] Guo S, Wang T, Chen J, Wu L, Gao P, Zhu D. Dynamic expression of proteins associated with adventitial remodeling in adventitial fibroblasts from spontaneously hypertensive rats. Acta Pharmacol Sin. 2010; 31(10): 1312-8.

[7] Damazo AS, Sampaio AL, Nakata CM, Flower RJ, Perretti M, Oliani SM. Endogenous annexin A1 counter-regulates bleomycin-induced lung fibrosis. BMC Immunol. 2011; 12(1): 59.

[8] Jia Y, Morand EF, Song W, Cheng Q, Stewart A, Yang YH. Regulation of lung fibroblast activation by annexin A1. J Cell Physiol. 2013; 228(July): 476-84.

[9] Trentin PG, T Ferreira TP, Arantes a CS, Ciambarella BT, Cordeiro RSB, Flower RJ, et al. Annexin A1-mimetic peptide controls the inflammatory and fibrotic effects of silica particles in mice. Br J Pharmacol. 2015.

[10] Neymeyer H, Labes R, Reverte V, Saez F, Stroh T, Dathe C, et al. Activation of annexin A1 signalling in renal fibroblasts exerts antifibrotic effects. Acta Physiol. 2015; 215(3): 144-58.

[11] Xue M, Jackson CJ. Extracellular Matrix Reorganization During Wound Healing and Its Impact on Abnormal Scarring. Adv Wound Care. 2015; 4(3): 119-36.

[12] Del Gaudio P, De Cicco F, Aquino RP, Picerno P, Russo P, Dal Piaz F, et al. Evaluation of in situ injectable hydrogels as controlled release device for ANXA1 derived peptide in wound healing. Carbohydr Polym. 2015; 115: 629-33.

[13] Dai T, Kharkwal GB, Tanaka M, Huang Y-Y, Bil de Arce VJ, Hamblin MR. Animal models of external traumatic wound infections. Virulence. 2011; 2(4): 296-315.

[14] Rutala WA, Weber DJ. Uses of inorganic hypochlorite (bleach) in health-care facilities. Clin Microbiol Rev. 1997; 10(4): 597-610.

[15] Fukuzaki S. Mechanisms of actions of sodium hypochlorite in cleaning and disinfection processes. Biocontrol Sci. 2006; 11(4): 147-57.

[16] Ono Tomoko, Koji Yaamashita, Tomomi Murayama TS. Microbicidal effect of a weak acid hypochlorus solution on various microrganisms.

[17] Bryce G, O’Donnell D, Ready D, Ng Y ling, Pratten J, Gulabivala K. Contemporary Root Canal Irrigants Are Able to Disrupt and Eradicate Single- and Dual-Species Biofilms. J Endod. 2009; 35(9): 1243-8.

[18] Fader RC, Maurer A, Stein MD, Abston S, Herndon DN. Sodium hypochlorite decontamination of split-thickness cadaveric skin infected with bacteria and yeast with subsequent isolation and growth of basal cells to confluency in tissue culture. Antimicrob Agents Chemother. 1983; 24(2): 181-5.

[19] Cotter JL, Fader RC, Lilley C, Herndon DN. Chemical parameters, antimicrobial activities, and tissue toxicity of 0.1 and 0.5\% sodium hypochlorite solutions. Antimicrob Agents Chemother. 1985; 28(1): 118-22.

[20] Coetzee E, Whitelaw A, Kahn D, Rode H. The use of topical, un-buffered sodium hypochlorite in the management of burn wound infection. Burns. 2012; 38(4): 529-33.

[21] Carmen R Michaud, Jing Qin, William R Elkins, Alfonso S Gozalo. Comparison of 3 Topical Treatments against Ulcerative Dermatitis in Mice with a C57BL/6 Background. Comp Med. 2016; 66(2): 100-4.

[22] Cambiaso-Daniel Janos et al. Topical Antimicrobials in Burn Care: Part I - Topical Antiseptics. Physiol Behav. 2017; 176(1): 139-48. 
[23] Wongkietkachorn A, Surakunprapha P, Wittayapairoch J, Wongkietkachorn N, Wongkietkachorn S. The use of hypochlorous acid lavage to treat infected cavity wounds. Plast Reconstr Surg - Glob Open. 2020; 1-3.

[24] Severing AL, Rembe JD, Koester V, Stuermer EK. Safety and efficacy profiles of different commercial sodium hypochlorite/hypochlorous acid solutions (NaClO/HClO): Antimicrobial efficacy, cytotoxic impact and physicochemical parameters in vitro. J Antimicrob Chemother. 2019; 74(2): 365-72.

[25] Huttunen M, Aalto ML, Harvima RJ, Horsmanheimo M, Harvima IT. Alterations in mast cells showing tryptase and chymase activity in epithelializating and chronic wounds. Exp Dermatol. 2000; 9(4): 258-65.

[26] Eroglu O, Deniz T, Kisa U, Comu FM, Kaygusuz S, Kocak OM. The effect of different types of honey on healing infected wounds. J Wound Care. 2018; 27(Sup10): S18-25.

[27] Essner MD, Javed A, Eleazer PD. Effect of sodium hypochlorite on human pulp cells: An in vitro study. Oral Surgery, Oral Med Oral Pathol Oral Radiol Endodontology. 2011; 112(5): 662-6.

[28] Leoni G, Alam A, Neumann P, Lambeth JD, Cheng G, Mccoy J, et al. Orchestrate Epithelial Repair. 2013; 123(1).

[29] Bufe B, Zufall F. The sensing of bacteria: emerging principles for the detection of signal sequences by formyl peptide receptors. Biomol Concepts. 2016.

[30] Weiß E, Kretschmer D. Formyl-Peptide Receptors in Infection, Inflammation, and Cancer. Trends Immunol. 2018; 39(10): 815-29.

[31] Liu M, Chen K, Yoshimura T, Liu Y, Gong W, Le Y, et al. Formylpeptide receptors mediate rapid neutrophil mobilization to accelerate wound healing. PLoS One. 2014; 9(3): 1-7.

[32] Barrientos S, Stojadinovic O, Golinko MS, Brem H, Tomic-Canic M. PERSPECTIVE ARTICLE: Growth factors and cytokines in wound healing. Wound Repair Regen. 2008; 16(5): 585-601.

[33] Kubo H, Hayashi T, Ago K, Ago M, Kanekura T, Ogata M. Temporal expression of wound healing-related genes in skin burn injury. Leg Med. 2014; 16(1): 8-13.

[34] Lee K, Lee B, Lee M-H, Kim B, Chinannai KS, Ham I, et al. Effect of Ampelopsis Radix on wound healing in scalded rats. BMC Complement Altern Med. 2015; 15(1): 213.

[35] Jiang Y, Lu S. Three-dimensional insights into dermal tissue as a cue for cellular behavior. Burns. 2014; 40(2): 191-9.

[36] Leung TH, Zhang LF, Wang J, Ning S, Knox SJ, Kim SK. Topical hypochlorite ameliorates NF- $\kappa$ B-mediated skin diseases in mice. J Clin Invest. 2013; 123(12): 5361-70. 